\title{
When to start treatment? Dilemma illustrated by a paediatric case of extensively drug-resistant tuberculosis of the central nervous system
}

\author{
R M S Gigi, ${ }^{1}$ MMed (Zurich); H L Rieder, ${ }^{2}$ MD, MPH Epidemiology; N Padayatchi, ${ }^{3} \mathrm{PhD}$ \\ ${ }^{1}$ Faculty of Medicine, University of Zurich, Switzerland \\ ${ }^{2}$ Biostatistics and Prevention Institute, University of Zurich, Switzerland \\ ${ }^{3}$ South African Medical Research Council HIV-TB Pathogenesis and Treatment Research Unit, Centre for the AIDS Programme of Research in \\ South Africa (CAPRISA), University of KwaZulu-Natal, Durban, South Africa
}

Corresponding author: R M S Gigi (ranjana.gigi@gmail.com)

\begin{abstract}
An HIV-positive mother infected her daughter with extensively drug-resistant Mycobacterium tuberculosis. Despite adhering to the then current guidelines for prevention, the infant was diagnosed with extensively drug-resistant pulmonary tuberculosis at the age of 4 months and developed tuberculous meningitis. After a short delay, appropriate treatment was initiated, followed by an inhospital stay at a specialised hospital. The infant became generally well, but had delayed neurological development. Secondary hydrocephalus due to tuberculous meningitis required a ventriculoperitoneal shunt. After 2 years of microbiologically and clinically effective tuberculosis treatment and several shunt complications, the HIV-negative child died at the age of 28 months - with radiological signs of a shunt infection. The reason for the fatal outcome was probably related to inadequate risk reduction of airborne mother-to-child transmission, inappropriate chemoprophylaxis and delayed initiation of adequate treatment.
\end{abstract}

S Afr Med J 2020;110(9):846-849. https://doi.org/10.7196/SAMJ.2020.v110i9.14567

Young children who become infected with Mycobacterium tuberculosis are at high risk of developing tuberculous meningitis, one of the most lethal forms of tuberculosis, particularly if appropriate treatment is delayed. ${ }^{[1]}$ Currently, there are no guidelines or evidence-based options for primary prophylaxis for children exposed to extensively drug-resistant tuberculosis (XDR-TB).

South Africa (SA) has one of the world's highest tuberculosis incidence rates ${ }^{[2]}$ and KwaZulu-Natal Province one of the world's worst epidemics of XDR-TB. ${ }^{[3]}$

We report the case of a mother-child pair with XDR-TB from this setting, which ended fatally in both patients, despite what appeared to be appropriate chemotherapy with an effective regimen of antituberculosis drugs adapted to the drug susceptibility pattern of their M. tuberculosis strain.

The patient was identified in an observational study. The information was extracted from the patient's records and family visits in 2016 and 2017.

\section{Case report}

A 34-year-old HIV-positive mother, treated with a standard antiretroviral drug regimen, was successfully treated for tuberculosis with first-line drugs in 2005. She developed a second episode of pulmonary tuberculosis in 2007. The resistance of the strain to previously never-used antituberculosis medications suggested XDR reinfection disease. The drug-susceptibility profile (resistance to isoniazid, rifampicin, ethambutol, ciprofloxacin, kanamycin and streptomycin) guided her treatment. Sputum specimens remained culture-positive before and after the uneventful birth of her daughter in 2010, despite reported good treatment adherence.

The child, HIV-uninfected and with unknown bacillus CalmetteGuérin vaccination status, received primary isoniazid prophylaxis for
3 months, in line with the national tuberculosis guidelines. ${ }^{[4]}$ At birth, a gastric lavage specimen was negative for $M$. tuberculosis, a chest radiograph was normal, and no evidence of congenital tuberculosis was present. The child was living with her mother and relatives in a township house without implementation of any specific infectioncontrol measures. No other member of the household was diagnosed with tuberculosis.

At the age of 4 months, the infant presented with right upper-lobe radiographic consolidation, interpreted as pneumonia. A gastric lavage specimen yielded $M$. tuberculosis, and first-line antituberculosis treatment was started (Fig. 1). Two weeks later, the isolate showed resistance to isoniazid and rifampicin by the GenoType MTBDRplus assay (Hain Lifescience, Germany), but the laboratory reported only 'resistant', without specifying the mutations. Treatment was changed to a regimen containing capreomycin, pyrazinamide, terizidone, moxifloxacin and para-aminosalicylic acid. The child was then hospitalised. The gastric lavage was repeated and yielded an M. tuberculosis isolate phenotypically also resistant to ofloxacin, kanamycin and streptomycin. Ethionamide was added to the regimen.

The infant was in a good general condition, but vomited repeatedly and became pyrexial, which in retrospect might have been the first signs of tuberculous meningitis. Amoxicillin/clavulanate was administered. At the age of 6 months, she had the first seizure and a bulging fontanelle. The sterile cerebrospinal fluid (CSF) showed slightly elevated proteins of $1.05 \mathrm{~g} / \mathrm{L}$, low glucose of $1.4 \mathrm{mmol} / \mathrm{L}$, polymorphs 14 cells $/ \mu \mathrm{L}$ and lymphocytes 96 cells $/ \mu \mathrm{L}$. Carbamazepine, ceftriaxone and a corticosteroid were administered. The infant was irritable, febrile and continued vomiting. Three weeks later, high-dose isoniazid and clofazimine were added to the regimen, as tuberculous meningitis was suspected. At 7 months 
of age, a computed tomography (CT) scan was interpreted as showing multiple tuberculomas around the right ventricle and both parietal lobes, meningeal enhancement and early hydrocephalus, consolidating the diagnosis of tuberculous meningitis. She was deemed unsuitable for a ventriculoperitoneal (VP) shunt, given the compensated hydrocephalus. The corticosteroid dose was tapered. During the subsequent months of hospitalisation, she vomited repeatedly, was pyrexial and irritable, had delayed motor development and a decreased body tone. Amoxicillin/clavulanate and para-aminosalicylic acid, but not ethionamide, were discontinued owing to concerns of medication-associated vomiting and diarrhoea. During this time, the child lost weight, catching up only months later (Fig. 2). The M. tuberculosis culture of gastric fluid converted to negative at the age of 8 months ( 3 months on XDR-TB treatment). Another seizure episode followed 2 months later. After 8 months on XDR-TB treatment, capreomycin was discontinued, and she was discharged from hospital in a fairly good general condition, with persistent neurodevelopmental delay. Her lungs were clinically and radiographically clear. High-dose isoniazid, pyrazinamide, terizidone, ethionamide, moxifloxacin, clofazimine and carbamazepine were continued at home under the care of her grandmother, with monthly outpatient follow-up. At the age of 16 months, she presented with a repeatedly stiff body and increased spasticity. The now non-compensated hydrocephalus required a VP shunt. The CSF was unremarkable during the implantation. The CT scan showed calcifying tuberculomas.

During the following months the child was well, with clinically clear lungs, but remained spastic with occasional seizures at home, and a general developmental delay of 1 year.

At the age of 26 months, she presented with increased seizure frequency and irritability, fever and neck stiffness. The CT scan showed an obstructive hydrocephalus attributed to a blocked shunt,

\begin{tabular}{|c|c|c|}
\hline Isoniazid & High dose \\
Rifampicin & \\
Pyrazinamide \\
Terizidone
\end{tabular}

Fig. 1. Treatment of the child with antituberculosis drugs by age (months).

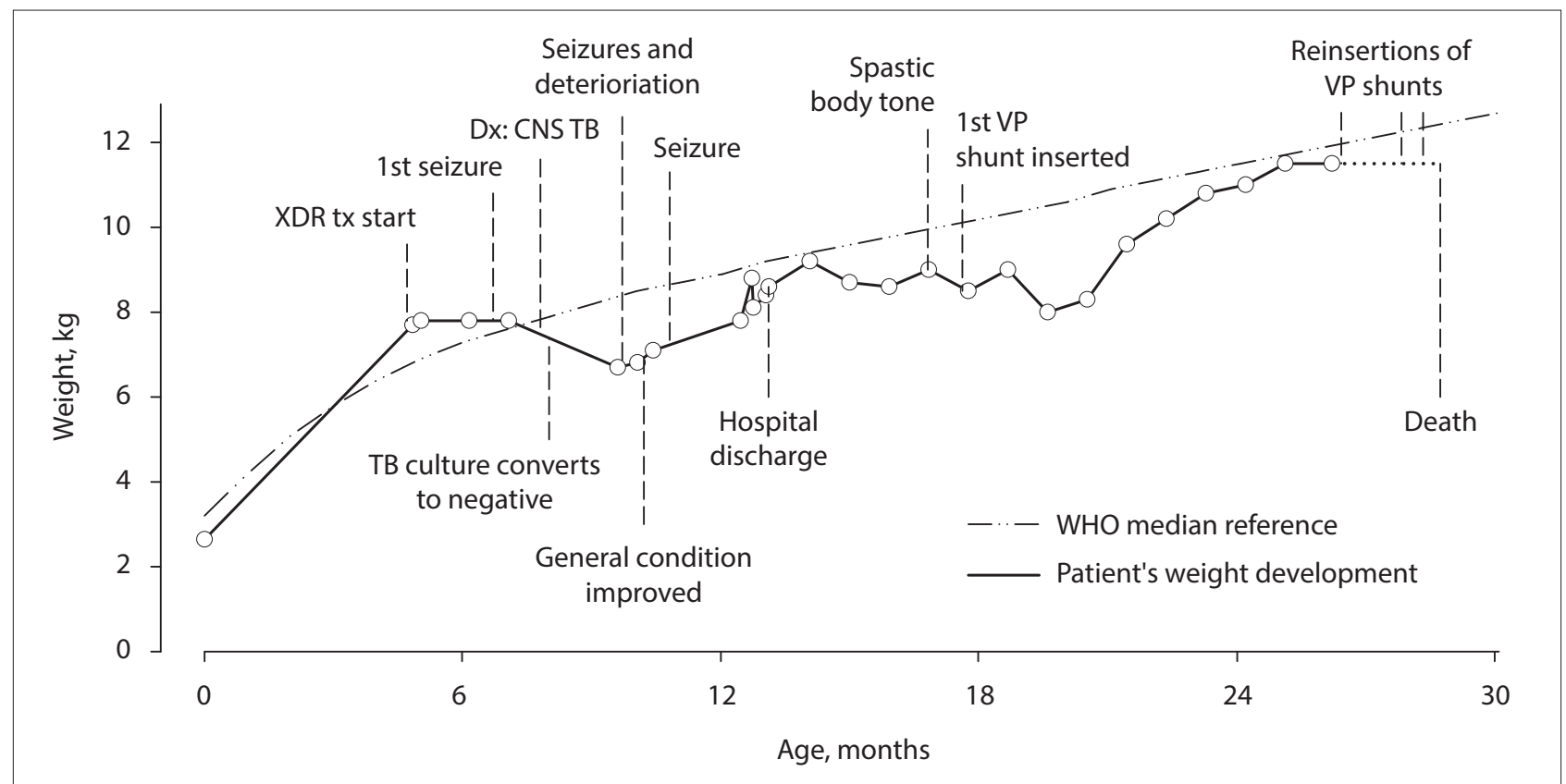

Fig. 2. Weight ( $\mathrm{kg}$ ) by age (months) of the child from birth to death (solid line) compared with the WHO reference median weight by age for girls (dot-dot-dashed line) ${ }^{[15]}$ Circles are points of actual measurements. $(X D R=$ extensively drug resistant; $t x=$ treatment; $D x=$ diagnosis; $C N S=$ central nervous system; $T B=$ tuberculosis; $V P=$ ventriculoperitoneal $; W H O=$ World Health Organization.$)$ 
requiring revision. Moxifloxacin was changed to levofloxacin for unknown reasons. One month later she presented again with fever, neck stiffness and lethargy. The CT scan showed that ventricle dilatation had increased. The shunt was removed, and an external ventricular drain was placed before inserting a new VP shunt when the patient was 27 months of age. One week after discharge, the child was readmitted with vomiting, seizures, fever and irritability. The magnetic resonance imaging (MRI) findings at the age of 28 months were interpreted as tuberculous meningitis and ependymitis with obstructive hydrocephalus due to a non-functioning VP shunt, which was again successfully revised. The CSF showed almost normal polymorph and lymphocyte counts of 2 cells $/ \mu \mathrm{L}$ and 6 cells $/ \mu \mathrm{L}$, respectively, and was microbiologically sterile. The last CT scan of the brain at the age of 28 months showed a functioning VP shunt with enhancement of the meninges and ventricles. The previous VP shunt tract indicated ventriculitis and inflammation, and signs of previously disseminated tuberculosis with residual calcifying tuberculomas. The anti-epileptic medication was changed from carbamazepine to sodium valproate. A day later the child's mother, who was very ill, died as an inpatient owing to XDR-TB treatment failure, with dyspnoea, cyanosis and general malaise. Six days later (24 months on XDR-TB treatment), at the age of 28 months, the child died at a regional hospital, with persistent neurological symptoms consistent with a hydrocephalus and most likely a VP shunt infection. No autopsy was performed.

\section{Discussion}

In this case, transmission of XDR-TB bacilli from mother to child was not prevented. No specific measures to lower the risk of airborne transmission from mother to child are known to have been taken. There are no consistent recommendations to reduce airborne transmission from mothers with infectious drug-resistant tuberculosis to their newborns. Separation of mother and infant and masks for the mother are possible solutions. ${ }^{[5,6]}$ In a low-income setting, it can be challenging to separate children from their mother and it is generally discouraged. Providing the mother with masks to wear during close contact with the child would have been feasible, but the effectiveness of non-fitted surgical masks in preventing transmission of droplet nuclei is largely unproven..$^{[7]}$

Prophylactic isoniazid treatment was given for only 3 months, whereas the guidelines recommend 6 months of treatment. However, it is unlikely that the reason for prophylaxis failure can be attributed to the treatment duration; it is most likely due to the failure of isoniazid to protect the child initially, allowing successful transmission while the child was on primary isoniazid prophylaxis.

The primary isoniazid chemoprophylaxis was possibly ineffective, but evidence for a more appropriate regimen is lacking. Knowledge about the genetic mutation that causes isonazid resistance (inhA promoter gene or $k a t G$ gene) is an insufficient basis for the decision to give normal- or high-dose isoniazid, as mutations in the kat $G$ gene can cause low-, middle- or high-level resistance to isoniazid. ${ }^{\left[{ }^{[8]}\right.}$ Some experience with primary fluoroquinolone chemoprophylaxis is available from children exposed to multidrug-resistant tuberculosis (MDR-TB) ${ }^{[9]}$ Any benefit of fluoroquinolones as primary chemoprophylaxis for persons exposed to XDR-TB is likely to be poorer than for MDR-TB. Reluctance to initiate potentially highly toxic drugs in an infant with no signs of tuberculosis, as required for primary chemoprophylaxis for drug-resistant tuberculosis exposure, is a comprehensible rationale.

Although the child was given XDR-TB treatment shortly after the first symptoms, she possibly died of the sequelae of meningitis (hydrocephalus with multiple VP shunt complications) after 2 years of treatment with a microbiologically appropriate (culture conversion of gastric lavage specimens) and clinically effective (weight gain and disappearance of inflammatory signs in the CSF) XDR-TB regimen. The underlying reason for the child developing XDR-TB (after strain acquisition, most probably from her mother) and the fatal outcome is most likely attributable to primary prevention failure with ensuing dissemination. This case highlights why early diagnosis with prompt initiation of appropriate treatment is so critical. Fatality from tuberculous meningitis rises from $5 \%$ to $80 \%$ when treatment is delayed for 5 weeks. ${ }^{[1]}$ Appropriate treatment was started later than it could and should have been, even if the delay was not very long.

When the child had a compensated hydrocephalus, only corticosteroids were administered as a medical therapy. Adjuvant furosemide and acetazolamide treatment for compensated hydrocephalus in children with tuberculous meningitis have been shown to have an effect on lowering of the intracranial pressure, but no effect on mortality or disability. ${ }^{[10]}$ In this case, it is questionable whether adding these drugs would have had any effect on the outcome. Furthermore, these drugs were not tested in XDR-TB meningitis and in combination with corticosteroids.

Only a few reports have been published of children with central nervous system XDR-TB. While there are publications on poor outcomes of pre-XDR-TB and MDR-TB of the central nervous system, ${ }^{[11,12]}$ there is also ground for some optimism demonstrated by the case of a 4-year-old girl in SA. ${ }^{[13]}$ She had delayed treatment initiation and early onset of neurological symptoms, but recovered fully from XDR-TB meningitis after 18 months of adequate treatment. ${ }^{[13]}$

Several studies showed a positive effect of newer drugs, delamanid and bedaquiline, on the treatment success of XDR-TB, but insufficient data are available on their safety in children, and the discussion for their use as primary prophylaxis for high-risk infants has to our knowledge not yet begun. ${ }^{[14]}$ In the time and context of this case, the newer drugs were not or scarcely available in SA.

It is long known that early adequate treatment of tuberculosis can prevent the deleterious effects of dissemination, but it contrasts with our ignorance about effective prevention for complex drugresistant tuberculosis. This child's case illustrates how important it is to act swiftly.

\section{Teaching points}

- If a neonate is exposed to XDR-TB, very frequent clinical assessments and measures to lower the risk of airborne transmission are mandatory. This allows possible prevention and early detection of clinical manifestations that require an aggressive approach to diagnosis and treatment of tuberculosis, if indicated.

- Chemoprophylaxis must be administered according to the latest guidelines, in consultation with a specialised facility.

- If a mother has XDR-TB, the child thought to have tuberculosis must be treated for XDR-TB - otherwise valuable time is wasted, potentially resulting in a disastrous outcome.

- The decisiveness of the approach must be adjusted to the infectivity potential of the index case.

\section{Declaration. None.}

\section{Acknowledgements. None.}

Author contributions. RMSG: main author, led the search for the medical records and drafted the manuscript; HLR: contributed to the discussion and writing of the manuscript; NP: supervised the search for the medical records on site and contributed to the writing of the report.

Funding. None.

Conflicts of interest. None. 
1. Thwaites G. Tuberculous meningitis. Medicine 2013;41(12):683-685. https://doi.org/10.1016/j. mpmed.2013.09.006

2. World Health Organization. Global Tuberculosis Report 2017. Geneva: WHO, 2017.

3. Nelson KN, Shah NS, Mathema B, et al. Spatial patterns of extensively drug-resistant tuberculosis transmission in KwaZulu-Natal, South Africa. J Infect Dis 2018;218(12):1964-1973. https://doi. org/10.1093/infdis/jiy394

4. Moore DP, Schaaf HS, Nuttall J, Marais BJ. Childhood tuberculosis guidelines of the Southern African Society for Paediatric Infectious Diseases. South Afr J Epidemiol Infect 2009;24(3):57-68. https://doi. Society for Paediatric Infectious Dise
org $/ 10.1080 / 10158782.2009 .11441353$

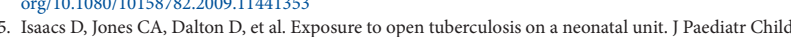
5. Isaacs D, Jones CA, Dalton D, et al. Exposure to open tuberculosis on a neona
Health 2006;42(9):557-559. https://doi.org/10.1111/j.1440-1754.2006.00923.x

6. Charney W, Fisher J, Ishida C. The inefficiency of surgical masks for protection against droplet nuclei Charney W, Fisher J, Ishida C. The inefficiency or
tuberculosis. J Occup Med 1991;33(9):943-944.

tuberculosis. J Occup Med 1991;33(9):943-944.
7. Hodous TK, Coffey CC. The role of respiratory protective devices in the control of tuberculosis. Occup Med 1994;9(4):631-657.

8. Böttger EC. The ins and outs of Mycobacterium tuberculosis drug susceptibility testing. Clin Microbiol 8. Böttger EC. The ins and outs of Mycobacterium tuberculosis drug susceptibility
Infect 2011;17(8):1128-1134. https://doi.org/10.1111/j.1469-0691.2011.03551

Infect 2011;17(8):1128-1134. https://doi.org/10.1111/j.1469-0691.2011.03551.x
9. Seddon JA, Hesseling AC, Finlayson H, et al. Preventive therapy for child contacts of multidrug9. Seddon JA, Hesseling AC, Finlayson $\mathrm{H}$, et al. Preventive therapy for child contacts of multidrug-
resistant tuberculosis: A prospective cohort study. Clin Infect Dis 2013;57(12):1676-1684. https://doi. org/10.1093/cid/cit655
10. Schoeman J, Donald P, van Zyl L, Keet M, Wait J. Tuberculous hydrocephalus: Comparison of different treatments with regard to ICP, ventricular size and clinical outcome. Dev Med Child Neurol 1991;33(5):396-405. https://doi.org/10.1111/j.1469-8749.1991.tb14899.x

1. Katragkou A, Antachopoulos C, Hatziagorou E, Sdougka M, Roilides E, Tsanakas J. Drug-resistant tuberculosis in two children in Greece: Report of the first extensively drug-resistant case. Eur J Pediatr 2013;172(4):563-567. https://doi.org/10.1007/s00431-012-1811-8

2. Padayatchi N, Bamber S, Dawood H, Bobat R. Multidrug-resistant tuberculous meningitis in children in Durban, South Africa. Pediatr Infect Dis J 2006;25(2):147-150. https://doi.org/10.1097/01. children in Durban, South Africa. Pediatr Infect Dis 2 2006;25(2):147-150. https://doi.org/10.1097/01. inf.0000199314.88063.4c

. Alsleben N, Garcia-Prats AJ, Hesseling AC, Willemse M, Donald PR, Schaaf HS. Successful treatment of a child with extensively drug-resistant tuberculous meningitis. J Pediatric Infect Dis Soc 2015;4(3):e41-e44. https://doi.org/10.1093/jpids/piul20

14. D’Ambrosio L, Centis R, Tiberi S, et al. Delamanid and bedaquiline to treat multidrug-resistant and extensively drug-resistant tuberculosis in children: A systematic review. J Thorac Dis 2017;9(7):2093-2101. https://doi.org/10.21037/jtd.2017.06.16

15. World Health Organization. Child Growth Standards. Simplified Field Tables. Weight-for-age Girls. Birth to 5 Years. Geneva: WHO, 2018

Accepted 14 April 2020 